\title{
Application of caspofungin in China compared with amphotericin $B$ and fluconazole
}

\author{
This article was published in the following Dove Press journal: \\ Therapeutics and Clinical Risk Management \\ 10 September 2014 \\ Number of times this article has been viewed
}

\section{Chunyu Zhang' \\ Jiaoying Cheng ${ }^{2}$ \\ Yan Jiang ${ }^{3}$ \\ Junyang $\mathrm{Liu}^{4}$}

'Department of Health Reform and Development, China-Japan Friendship Hospital, Beijing, People's Republic of China; ${ }^{2}$ Department of Obstetrics and Gynecology, China-Japan Friendship Hospital, Beijing, People's Republic of China; ${ }^{3}$ National Management Center of 12320 Health Hotline, Chinese Center for Disease Control and Prevention, Beijing, People's Republic of China; ${ }^{4}$ Department of Pharmacy, China-Japan Friendship Hospital, Beijing, People's Republic of China

Correspondence: Chunyu Zhang Department of Health Reform and Development, China-Japan Friendship Hospital, No 2, Yinghua East Road, Beijing 100029, People's Republic of China

Tel +86 I0 84205990

Email zcy_fish@sina.com

\begin{abstract}
Fungal infection has increased in the past 2 decades in China. There are three classes of antifungal drugs, polyenes, azoles, and echinocandins, that are applied frequently in China. Caspofungin, which disrupts the fungal cell wall glucan formation through inhibiting the enzyme $1,3-\beta$-glucan synthase, is one of the echinocandins. According to the results of clinical practices applied in China, caspofungin has shown to be superior to the other two classes of antifungal drugs, due to its efficacy in treating fungal infection (15\% superior to fluconazole); fewer adverse events such as infusion-related reaction, hepatic dysfunction, and vomiting $(25 \%-50 \%$ lower incidence rate); rapid resolution of symptoms (about 3 days quicker than amphotericin B); and absence of antagonism in combination with other antifungal drugs. However, caspofungin will remain as a second-line antifungal drug in the near future because of its high price and the policy of health insurance reimbursement in China.
\end{abstract}

Keywords: fungal infection, caspofungin, efficacy, adverse event

\section{Introduction}

The past 2 decades have witnessed a steady increase in the incidence of fungal infections due to the application of immunosuppressants and broad-spectrum antibiotics in China. ${ }^{1,2}$ Fungal infections are frequently observed in immunocompromised patients. These include patients who have acquired immunodeficiency syndrome (AIDS), those receiving chemotherapy with malignancies and hematologic diseases, and those with other serious underlying illnesses. ${ }^{3-5}$ Candida and invasive aspergillosis, which are typical fungi, are responsible for serious levels of morbidity and mortality. It is reported that the mortality rate of invasive aspergillosis infection is $>30 \%$ in China, similar with other countries and districts. ${ }^{6-8}$

More and more Chinese physicians and health care managers are paying attention to the treatment of fungal infection. Although there is good progress in treating fungal infection, challenges still exist. First of all, much research has shown that $8 \%-15 \%$ of nosocomial infections were attributed primarily to fungal infection. ${ }^{9}$ Candida and invasive aspergillosis were most common. ${ }^{10}$ There are cases showing Candida and invasive aspergillosis resistant to amphotericin B and azole derivatives. ${ }^{11-13}$ Secondly, there is no gold rule to diagnose fungal infection, due to the immature fungal diagnosis method. As a result, the reported incidence of fungal infection is lower than the real incidence, the dangers of fungal infection are not realized by many physicians, and empirical therapy is adopted without laboratory diagnosis. ${ }^{14,15}$ Thirdly, the incidence of fungal infection has increased rapidly and is ahead of the speed of the research of diagnosis and treatment. ${ }^{16}$ Twenty percent to $40 \%$ of patients have undergone organ transplant and $90 \%$ of AIDS patients were infected by fungi. ${ }^{9}$ 


\section{The current treatment options for fungal infections}

There are three classes of antifungal drugs, polyenes, azoles, and echinocandins, that are most frequently used worldwide nowadays (Table 1).

Polyene, eg, amphotericin B, has potent antifungal action and broad antifungal activity against the majority of yeasts, including Candida and molds, through changing the permeability of the fungal cell wall. There are reports that the effective rate of amphotericin B in treating invasive fungal infection was about $60 \%$. There were no significant changes in serum creatinine and potassium levels. ${ }^{17} \mathrm{How}-$ ever, amphotericin B in treating fungal infection is not the first choice, due to its severe adverse events, such as nephrotoxicity, infusion-related reaction, fever, and vomiting. ${ }^{18-20}$ Amphotericin B is demonstrated to be poorly tolerated. It is difficult to administer for a sufficiently long duration.

Azoles such as itraconazole, voriconazole, fluconazole, and posaconazole are the main triazole antifungal drugs that inhibit the enzyme lanosterol demethylase by blocking the biosynthesis of ergosterol. ${ }^{21}$ Fluconazole is used as a first-line therapy for Candida, which is responsible for $80 \%$ of nosocomial fungal infection. However, the number of susceptible species that are resistant to azoles is increasing due to their extensive use..$^{22,23}$

Echinocandins include caspofungin, micafungin, and anidulafungin. These antifungi inhibit the enzyme 1,3- $\beta$ glucan synthase, then result in disruption of cell wall glucan formation. ${ }^{24}$ Caspofungin is indicated for the treatment of Candida and invasive aspergillosis. Micafugin does well in treating Candida. Anidulafugin is an antifungal drug for the majority of Candida, including those with resistance to fluconazole. Compared with the other two classes of antifungal drugs, echinocandins have a broader spectrum of antifungal activity and fewer adverse events. There are reports that the incidence of liver and kidney dysfunction was $3.8 \%$, which is significantly lower than that of amphotericin B
(13.4\%), when treating invasive aspergillosis in an aged population. ${ }^{25,26}$ Meanwhile, echinocandins offer relatively rapid resolution of symptoms but fewer or negligible interactions with other drugs. ${ }^{27,28}$

\section{Pharmacology of caspofungin}

Caspofungin is one of the echinocandin antifungal drugs, with a mechanism of action that targets a structural component of the fungal cell wall. Caspofungin is an inhibitor of $1,3-\beta-$ glucan synthesis, which is a structural component of the fungal cell wall responsible for maintaining integrity and rigidity but is absent from mammalian cells..$^{29,30}$ When $1,3-\beta$-glucan synthesis of a fungal cell wall is inhibited by caspofungin, the cellular contents will balloon out from the weakened cell wall because of the high osmotic pressure of the protoplast. The lysis of the cell will happen consequently. ${ }^{31}$

Poor absorption after oral administration $(<0.2 \%)$ limits use to the intravenous route. After intravenous injection, caspofungin highly binds with protein (about 96\%). Caspofungin is widely distributed in the body and is metabolized by the liver. It is degraded mainly in the liver by hydrolysis and $\mathrm{N}$-acetylation, and a small part of it is also degraded in the adrenals and spleen. ${ }^{32,33}$ The slow phase of degradation leads to a long terminal half-life. As a result, the $\beta$-phase half-life is $9-10$ hours. ${ }^{34}$ Spontaneous chemical degradation can be observed in caspofungin. It is reported that the average plasma clearance rate was about $10-12 \mathrm{~mL} / \mathrm{min} .{ }^{35}$ Results of radiolabel studies suggested that $35 \%$ of degradation products were in the bile and excreted through feces, $41 \%$ of those were excreted through urine, and only about $1.4 \%$ of residual drugs were excreted through urine without degradation. ${ }^{36} \mathrm{It}$ can be concluded from the radiolabel result that the liver is the most important metabolic organ for caspofungin, although some of the degradation products are excreted through skin. ${ }^{37}$ Plasma concentrations of caspofungin will slightly increase when renal failure patients are exposed to caspofungin. This phenomenon has nothing to do with renal excretion or

Table I The characteristics of the three main classes of antifungal drugs

\begin{tabular}{|c|c|c|c|c|}
\hline Type & Typical drugs & Pharmacology & Good points & Shortcoming \\
\hline Polyenes & Amphotericin B & $\begin{array}{l}\text { Change the permeability of } \\
\text { the fungal cell wall }\end{array}$ & $\begin{array}{l}\text { High effective rate of treating } \\
\text { invasive fungal infection }\end{array}$ & $\begin{array}{l}\text { Severe adverse } \\
\text { reaction }\end{array}$ \\
\hline Azoles & $\begin{array}{l}\text { Itraconazole, voriconazole, } \\
\text { fluconazole, posaconazole }\end{array}$ & $\begin{array}{l}\text { Inhibit the enzyme lanosterol } \\
\text { demethylase by blocking the } \\
\text { biosynthesis of ergosterol }\end{array}$ & $\begin{array}{l}\text { High effective rate of treating } \\
\text { Candida }\end{array}$ & Resistance \\
\hline Echinocandins & $\begin{array}{l}\text { Caspofungin, micafungin, } \\
\text { anidulafungin }\end{array}$ & $\begin{array}{l}\text { Disrupt the cell wall } \\
\text { formation in fungi by } \\
\text { inhibiting the enzyme I,3- } \beta \text { - } \\
\text { glucan synthase }\end{array}$ & $\begin{array}{l}\text { Broader spectrum of antifungal } \\
\text { activity, fewer adverse effects, } \\
\text { rapid resolution of symptoms, } \\
\text { fewer interactions }\end{array}$ & Expensive \\
\hline
\end{tabular}


plasma protein binding. ${ }^{38,39}$ For severe hepatic dysfunction patients, it is recommended that dosage should be reduced to $50 \%$ daily dose after a standard loading dose, although this recommendation is not backed by clinical data. ${ }^{40,41}$ Although when compared with young patients plasma concentrations of caspofungin in the aged patients increase slightly due to slower metabolism, dosage alteration is not needed. ${ }^{42}$

For caspofungin, drug interactions are few. A liver enzyme inducer will accelerate the metabolism of caspofungin in the liver, resulting in the decreased plasma concentration of caspofungin. Caspofungin and cyclosporin are documented interactions, resulting in raised plasma concentrations of caspofungin. There is no report about interaction with other antifungal drugs such as itraconazole and amphotericin B..$^{43,44}$

\section{The application of caspofungin in Chinese patients}

Fungal infections are frequently observed in immunocompromised patients, as mentioned. The majority of fungal infections are caused by Candida and refractory invasive aspergillosis. Caspofungin is the first echinocandin antifungal drug. Although there is no caspofungin made in China, its efficacy has been witnessed in clinical practice in China.

According to the Chinese guidelines for fungal infection treatment for immunocompromised patients, caspofungin is recommended to prevent invasive fungal infection until the status of immunocompromised alleviates. The prevention dosage is $50 \mathrm{mg}$ per day. For those patients who are proved to be infected by Candida through the blood culture results or who cannot tolerate other classes of antifungal drugs, when severe adverse events appear, caspofungin will be applied. As with other countries, the loading dosage is $70 \mathrm{mg}$, followed by $50 \mathrm{mg}$ daily. Only in moderate or severe hepatic dysfunction patients should caspofungin need be decreased to $35 \mathrm{mg}$. ${ }^{9,45}$

\section{The efficacy, safety, and tolerability of caspofungin in Chinese patients}

There is a clinical study where caspofungin was compared with fluconazole to evaluate its efficacy, safety, and tolerability in China. Thirty-six patients with systemic fungal infections in an intensive care unit were randomly assigned to receive caspofungin or fluconazole. The caspofungin group, which had 18 patients, was treated with caspofungin for 14 days $(70 \mathrm{mg}$ loading dose, followed by $50 \mathrm{mg}$ daily). The fluconazole group, which also had 18 patients, was treated with $50 \mathrm{mg}$ fluconazole daily for the whole 14 days. Efficacy and adverse events in the two groups were observed. The effective rate was $100 \%$ in the caspofungin group and $66.7 \%$ in the fluconazole group.
The incidence rate of adverse events in the caspofungin group was $22.2 \%$, while it was $72.2 \%$ in the fluconazole group. There were significant differences in the effective rates between the two groups $(P<0.05)$, and the incidence rate of adverse events in the caspofungin group was significantly lower than that in the fluconazole group $(P<0.05)$. This result suggested that caspofungin was superior to fluconazole in both efficacy and safety in the treatment of severe pulmonary fungal infection in an intensive care unit. ${ }^{46}$

Another comparative study of caspofungin and amphotericin $\mathrm{B}$ deoxycholate in invasive fungal infections was also conducted in hematologic malignancy patients. A total of 68 cases of hematologic malignancy patients had secondary invasive fungal infection. They were divided into two groups. Group 1, which had 35 patients, received amphotericin B treatment only (5 mg loading dose with an additional $5 \mathrm{mg}$ daily the following day), while group 2 , which had 33 patients, was given an intravenous injection of caspofungin $(70 \mathrm{mg}$ loading dose, followed by $50 \mathrm{mg}$ daily), followed by oral administration of intraconazole. Clinical symptoms (such as fever) in group 1, when treated with amphotericin B, disappeared slowly. The average time that obvious efficacy could be observed in the amphotericin B group was 7-10 days. The process of efficacy appearance of the caspofungin group (3-7 days) was quicker than in the amphotericin B group. Of a total of 35 cases in the amphotericin B group, 25 cases were effective, with the total effective rate of $71.42 \%$, and 25 of 33 cases in the caspofungin group were effective, with the effective rate of $75.75 \%$. Both of the two groups showed good clinical efficacy, and there was no significant difference between those two groups. However, the incidence of adverse effects in the amphotericin B group was significantly higher than in the caspofungin group. The incidence of infusionrelated reactions was $31.43 \%$ in the amphotericin B group and $6.06 \%$ in the caspofungin group. The incidence of damage to liver and renal function was $51.43 \%$ in the amphotericin B group and $18.18 \%$ in the caspofungin group. The incidence of serious hypokalemia was $60 \%$ in the amphotericin B group and $9.09 \%$ in the caspofungin group. There was no patient withdrawal until the whole treatment course was completed. ${ }^{47}$

There is also research about caspofungin carried out in aged patients. A total of 50 aged patients (all of them aged $>80$ years) were diagnosed with invasive fungal disease, including 16 laboratory-confirmed cases, 18 clinic-confirmed cases, and 16 probable cases. Forty-one of them were given caspofungin and nine of them were given fluconazole for 2-9 days, then turned to caspofungin due to poor improvement. The majority of those patients were given a $70 \mathrm{mg}$ loading dose 
then $50 \mathrm{mg}$ daily, except two patients who were found to have hepatic dysfunction before treatment and who were given a $70 \mathrm{mg}$ loading dose, which was then reduced to $35 \mathrm{mg}$ per day. The whole treatment course was 2-79 days. The average treatment course was $17.5 \pm 15.8$ days. The average effective rate was $74 \%$. The average effective rate for those nine patients who were treated with caspofungin substituted for fluconazole was $66.7 \%$. Common adverse events such as chills, fever, headache, nausea, vomit, and diarrhea were not observed. However, liver damage could be observed in the treatment course. Only five patients showed the advent of liver damage or aggravation of liver damage. One of the five patients was found to have a liver disorder before treatment. On the 26th day after treatment, liver damage appeared. On the 34th day after treatment, the basic disease was aggravated and the patient died from respiratory failure. The hepatic function of the remaining four patients continued aggravating, even though use of caspofungin was stopped. This result suggests that there is no significant relationship between caspofungin and liver damage that appeared in the course of treatment with caspofungin. Although one patient withdrew due to decreasing neutrophils, the others adhered to the whole treatment course. ${ }^{48}$

\section{Patient-focused perspectives}

Like other countries, the most commonly reported adverse events with caspofungin in China have included fever, infusion-related reaction, headache, nausea, elevation in liver transaminase levels, and histamine-type reaction. ${ }^{49,50}$ Although those adverse events exist, physicians clearly know how to avoid or reduce those adverse events. Caspofungin has shown high efficacy of treating the majority of fungal infections, especially those resistant to common antifungal drugs. In total, patients usually adhere to the whole treatment. According to a multicenter observational study in which caspofungin was applied to treat postsolid organ transplant invasive fungal disease in China, Germany, Italy, and the UK, no serious drugrelated adverse events were reported and caspofungin was well tolerated as caspofungin monotherapy or as combination therapy with other antifungal drugs. ${ }^{51}$ However, caspofungin is still not included in the list of health insurance reimbursements in most provinces of China. This means that patients pay by out-of-pocket if they want to use caspofungin. Due to its high price, caspofungin is still not used widely in China. It will be applied when the other two classes of antifungal drugs are proved useless or severe liver damage appears.

\section{Conclusion}

Fungal infection has attracted the attention of Chinese physicians. It is more frequently reported that the traditional antifungal drugs such as fluconazole and amphotericin B are resistant to fungal infection or have more adverse events, while caspofungin showed equivalent efficacy in treating fungal infection in Chinese clinical practice. The absence of antagonism and substantially fewer toxic effects in combination with other antifungal drugs will result in its broad use in the near future. Considering its high price, combination antifungal therapy could become a general feature for caspofungin.

\section{Disclosure}

The authors report no conflicts of interest in this work.

\section{References}

1. Yang H, Huang W. [Research progress of epidemiology and detection method for fungal infection]. Medical Recapitulate. 2013;19:2956-2970. Chinese.

2. Cheng J. [Clinical analysis of 120 patients with diabetes and tuberculosis merger fungal infection]. Chinese and Foreign Medical Research. 2013;11(22):31-32. Chinese.

3. Guo Z, Chen H, Lou X, He X, Liu X. [Acute myeloid leukemia invasive fungal infection caspofungin amphotericin B]. Zhonghua Yi Yuan Gan Ran Xue Za Zhi. 2013;23(14):3369-3371. Chinese.

4. Huang L, Tang X, Cai W, Lei C, Zheng F, Chen X. [Analysis of death causes of 345 eases with HIV/AIDS in Guangdong area]. Zhonghua Shi Yan He Lin Chuang Bing Du Xue Za Zhi. 2013;27(1):57-60. Chinese.

5. Wang J, Wang X. [Analysis on characteristics and prophylaxis of fluconazole of fungal infection in ICU]. China Modern Doctor. 2013;51(18): 158-160. Chinese.

6. Li F, Wu L, Cao B, Zhang Y, Li X, Liu Y. Surveillance of the prevalence, antibiotic susceptibility, and genotypic characterization of invasive candidiasis in a teaching hospital in China between 2006 to 2011. BMC Infect Dis. 2013;13(1):353.

7. Chen LY, Liao SY, Kuo SC, et al. Changes in the incidence of candidaemia during 2000-2008 in a tertiary medical centre in Northern Taiwan. J Hosp Infect. 2011;8(1):50-53.

8. Bassetti M, Merelli M, Righi E, et al. Epidemiology, species distribution, antifungal susceptibility, and outcome of candidemia across five sites in Italy and Spain. J Clin Microbiol. 2013;51(12):4167-4172.

9. Chinese Medical Association. [The guideline of diagnosis and treatment for invasive fungal infection patients]. Zhonghua Nei Ke Za Zhi. 2007; 46(11):960-966. Chinese.

10. Xing H, Sun D, Tian H. [Composition of hospital-acquired infections and community-acquired infections in a general hospital and analysis of drug susceptibility]. Zhonghua Yi Yuan Gan Ran Xue Za Zhi. 2013; 23(5):1142-1144. Chinese.

11. Liu Y, Liu Y. [Treatment outcomes of critically ill patients complicated with invasive fungal infections]. Zhonghua Yi Yuan Gan Ran Xue Za Zhi. 2013;23(18):4584-4586. Chinese.

12. Liu Y, Zhang H, Chen J, Dai Y, Liu T. [Species distribution and drug resistance in nosocomial infections with Candida]. Zhongguo Kang Sheng Su Za Zhi. 2013;38(6):473-475. Chinese.

13. Li Y, Xia Z, Li Z, Fan Q. [Distribution and drug resistance analysis on fungal infection in the urinary tract]. Xian dai Yu fang Yi xue. 2013; 40(7):1330-1335. Chinese.

14. Wang X, Wang B, Zhao C, Xi A, Liu R, Song J. [Clinical analysis on the diagnosis and treatment of AIDS with cryptococcal meningitis as the initial symptoms]. Chinese General Practice. 2012;15(36):4218-4221. Chinese.

15. Zhang X, Shi Z, Zhang H. [The analysis of deep fungal infection]. China Medical Herald. 2006;3(30):144-145. Chinese.

16. Sang H, Ni R, Liao W. [Current advances in the treatment of systemic fungal infection]. Yi хие Yan jiu Sheng xие Baо. 2003;16(1):44-47. Chinese. 
17. Kotani T, Takeuchi T, Makino S, et al. Efficacy and safety of liposomal amphotericin B for deep mycosis in patients with connective tissue disease. J Infect Chemother. 2013;19:691-697.

18. Adler-Moore J, Proffitt RT. AmBisome: liposomal formulation, structure, mechanism of action and pre-clinical experience. $J$ Antimicrob Chemother. 2002;49:21-30.

19. Herbrecht R, Denning DW, Patterson TF, et al. Voriconazole versus amphotericin B for primary therapy of invasive aspergillosis. $N \mathrm{Engl}$ J Med. 2002;347(6):408-415.

20. Mousset S, Buchheidt D, Heinz W, et al. Treatment of invasive fungal infections in cancer patients: updated recommendations of the Infectious Diseases Working Party (AGIHO) of the German Society of Hematology and Oncology (DGHO). Ann Hematol. 2014;93(1):13-32.

21. Onishi J, Meinz M, Thompson J, et al. Discovery of novel antifungal (1,3)-beta-D-glucan synthase inhibitors. Antimicrob Agents Chemother. 2000;44(2):368-377.

22. Linares CE, Giacomelli SR, Altenhofen D, Alves SH, Morsch VM, Schetinger MR. Fluconazole and amphotericin-B resistance are associated with increased catalase and superoxide dismutase activity in Candida albicans and Candida dubliniensis. Rev Soc Bras Med Trop. 2013;46(6):752-758.

23. Gomez-Lopez A, Forastiero A, Cendejas-Bueno E, et al. An invertebrate model to evaluate virulence in Aspergillus fumigatus: the role of azole resistance. Med Mycol. 2014;52(3):311-319.

24. Bartizal K, Abruzzo G, Trainor C, et al. In vitro antifungal activities and in vivo efficacies of 1,3-beta-D-glucan synthesis inhibitors L-671,329, L-646,991, tetrahydroechinocandin B, and L-687,781, a papulacandin. Antimicrob Agents Chemother. 1992;36(8):1648-1657.

25. Wang LL, Zhuge X, Xiao GH, Zhang Y. Hepatic safety caspofungin during treatment of invasive disease in elderly patients. Chin Med J (Engl). 2012;125(12):2240.

26. Paramythiotou E, Frantzeskaki F, Flevari A, Armaganidis A, Dimopoulos G. Invasive fungal infections in the ICU: how to approach, how to treat. Molecules. 2014;19(1):1085-119.

27. Méan M, Marchetti O, Calandra T. Bench-to-bedside review: Candida infections in the intensive care unit. Crit Care. 2008;12(1):204.

28. Kett DH, Shorr AF, Reboli AC, Reisman AL, Biswas P, Schlamm HT. Antidulafungin compared with fluconazole in severely ill patients with candidemia and other forms of invasive candidiasis: support for the 2009 IDSA treatment guidelines for candidiasis. Crit Care. 2011;15(5):R253.

29. Marco F, Pfaller MA, Messer SA, Jones RN. Activity of MK-099(L743,872), a new echinocandin compared with those of LY303366 and four other antifungal agents tested against blood stream isolates of Candida spp. Diagn Microbiol Infect Dis. 1998;32:33-37.

30. Debono M, Gordee RS. Antibiotics that inhibit fungal cell wall development. Annu Rev Microbiol. 1994;48:471-497.

31. Boucher HW, Groll AH, Chiou CC, Walsh TJ. Newer systemic antifungal agents: pharmacokinetics, safety and efficacy. Drugs. 2004;64(18): 1997-2020.

32. Denning DW. Echinocandin antifungal drugs. Lancet. 2003;362(9390): 1142-1151.

33. Chen SC, Slavin MA, Sorrell TC. Echinocandin antifungal drugs in fungal infections: a comparison. Drugs. 2011;71(1):11-41.

34. Migoya EM, Mistry GC, Stone JA, et al. Safety and pharmacokinetics of higher doses of caspofungin in healthy adult participants. J Clin Pharmacol. 2011;51(2):202-211.
35. Stone JA, Holland SD, Wichersham PJ, et al. Single- and multiple-dose pharmacokinetics of caspofungin in healthy men. Antimicrob Agents Chemother. 2002;46(3):739-745.

36. Stevens DA. Advances in systemic antifungal therapy. Clin Dermatol. 2012;30(6):657-661.

37. Badali H, Bonifaz A, Barrón-Tapia T, et al. Rhinocladiella aquaspersa, proven agent of verrucous skin infection and a novel type of chromoblastomycosis. Med Mycol. 2010;48(5):696-703.

38. Pasticci MB, Barchiesi F, Fallani S, et al. Clinical efficacy and tolerability of caspofungin in a renal transplant patient with Aspergillus flavus lung infection: case report. J Chemother. 2006;18(5):549-553.

39. Lilly CM, Welch VL, Mayer T, Ranauro P, Meisner J, Luke DR. Evaluation of intravenous voriconazole in patients with compromised renal function. BMC Infect Dis. 2013;16:13-14

40. van der Elst KC, Brüggemann RJ, Rodgers MG, Alffenaar JW. Plasma concentrations of caspofungin at two different dosage regimens in a patient with hepatic dysfunction. Transpl Infect Dis. 2012;14(4): 440-443.

41. Spriet I, Meyfroidt G, Maleux G, Verslype C, Willems L. The impact of a transjugular intrahepatic portosystemic shunt on the pharmacokinetics of caspofungin in a critically ill patient. Pharmacology. 2012;90(5-6): 247-250.

42. Scott LJ. Micafungin: a review of its use in the prophylaxis and treatment of invasive Candida infections. Drugs. 2012;72(16):2141-2165.

43. Del Poeta M, Cruz MC, Cardenas ME, Perfect JR, Heitman J. Synergistic antifungal activities of bafilomycin A(1), fluconazole, and the pneumocandin MK-0991/caspofungin acetate (L-743,873) with calcineurin inhibitors FK506 and L-685,818 against Cryptococcus neoformans. Antimicrob Agents Chemother. 2000;44(3):739-746.

44. Liu W, Li L, Sun Y, et al. Interaction of the echinocandin caspofungin with amphotericin B or voriconazole against Aspergillus biofilms in vitro. Antimicrob Agents Chemother. 2012;56(12):6414-6416.

45. Rüping MJ, Vehreschild JJ, Cornely OA. Antifungal treatment strategies in high risk patients. Mycoses. 2008;51 Suppl 2:46-51.

46. Zhu A, Xue L. [Caspofungin vs fluconazole in the treatment of severe pulmonary fungal infection in intensive care unit]. China Pharmacy. 2009;20(26):2047-2049. Chinese.

47. Luo H, Fu G, Zhang Y, Feng W, Hong P. [Efficacies of amphotericin B and caspofungin combined with intraconazole in treatment of invasive fungal infections inpatients with hematologic malignancies: a comparative study]. Zhonghua Yi Yuan Gan Ran Xue Za Zhi. 2013;23(22): 5530-5532. Chinese.

48. Wang L, Zhuge X, Xia GH, Zhang Y. [Analysis of efficacy and safety of caspofungin on treatment of invasive fungal disease in elderly patients]. Chin J Geriatr. 2013;32(12):1348-1350. Chinese.

49. Stone EA, Fung HB, Kirschenbaum HL. Caspofungin: an echinocandin antifungal agent. Clin Ther. 2002;24(3):351-377.

50. Yang W, Ou H. [Adverse effect of caspofungin and management in patients with invasive aspergillosis]. Journal of China Medical University. 2007;36(1):108-109. Chinese.

51. Winkler M, Pratschke J, Schulz U, et al. Caspofungin for post solid organ transplant invasive fungal disease: results of a retrospective observational study. Transpl Infect Dis. 2010;12(3):230-237.
Therapeutics and Clinical Risk Management

\section{Publish your work in this journal}

Therapeutics and Clinical Risk Management is an international, peerreviewed journal of clinical therapeutics and risk management, focusing on concise rapid reporting of clinical studies in all therapeutic areas, outcomes, safety, and programs for the effective, safe, and sustained use of medicines. This journal is indexed on PubMed Central, CAS,

\section{Dovepress}

EMBase, Scopus and the Elsevier Bibliographic databases. The manuscript management system is completely online and includes a very quick and fair peer-review system, which is all easy to use. Visit http://www.dovepress.com/testimonials.php to read real quotes from published authors. 